Document downloaded from:

http://hdl.handle.net/10251/100335

This paper must be cited as:

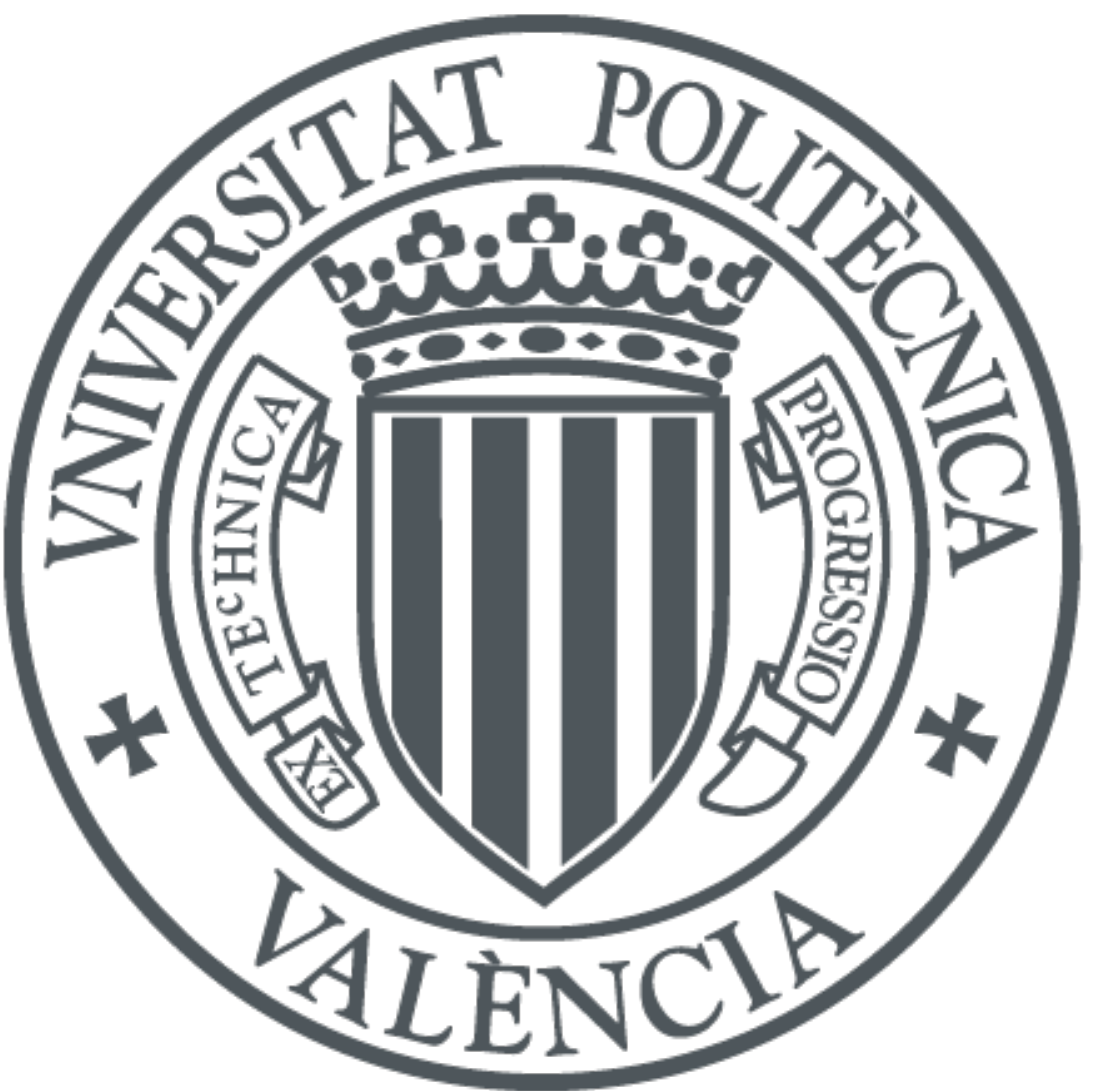

The final publication is available at

https://doi.org/10.1016/j.postharvbio.2016.11.006

Copyright Elsevier

Additional Information 


\title{
1 Astringency assessment of persimmon by hyperspectral imaging
}

\author{
2 Sandra Munera ${ }^{1(*)}$, Cristina Besada $^{2(*)}$, José Blasco $^{1}$, Sergio Cubero ${ }^{1}$, Alejandra Salvador ${ }^{2}$, Pau Talens ${ }^{3}$, \\ Nuria Aleixos ${ }^{4(* *)}$ \\ 4 \\ ${ }^{1)}$ Centro de Agroingeniería. Instituto Valenciano de Investigaciones Agrarias (IVIA). Ctra. Moncada-Náquera \\ Km 4.5, 46113, Moncada, Valencia (Spain) \\ ${ }^{2)}$ Centro de Tecnología Postcosecha. Instituto Valenciano de Investigaciones Agrarias (IVIA). Ctra. Moncada- \\ 8 Náquera Km 4.5, 46113, Moncada, Valencia (Spain) \\ $9 \quad{ }^{3)}$ Departamento de Tecnología de Alimentos. Universitat Politècnica de València. Camino de Vera, s/n, 46022 \\ 10 Valencia (Spain) \\ $11{ }^{4)}$ Departamento de Ingeniería Gráfica. Universitat Politècnica de València. Camino de Vera, s/n, 46022 \\ 12 Valencia (Spain) \\ * These authors contributed equally \\ **E-mail of the corresponding author: naleixos@dig.upv.es

\section{ABSTRACT}

One of the current challenges of persimmon postharvest research is the development of non-destructive methods that allow determination of the internal properties of the fruit, such as maturity, flesh firmness and astringency. This study evaluates the usefulness of hyperspectral imaging in the $460-1020 \mathrm{~nm}$ range as a non-destructive tool to achieve these aims in Persimmon cv. 'Rojo Brillante' which is an astringent cultivar. Fruit were harvested at three different stages of commercial maturity and exposed to different treatments of $\mathrm{CO}_{2}$ $\left(95 \% \mathrm{CO}_{2}-20^{\circ} \mathrm{C}-\right.$ from 0 to $24 \mathrm{~h}$ ) in order to obtain fruit with different levels of astringency. Partial Least Square (PLS) based methods were used to classify persimmon fruits by maturity and to predict flesh firmness from the average spectrum of each fruit. The results showed a $97.9 \%$ rate of correct maturity classification and an $\mathrm{R}_{\mathrm{P}}^{2}$ of 0.80 for firmness prediction with only five selected wavelengths. For astringency assessment, as our results showed that the soluble tannins that remain after $\mathrm{CO}_{2}$ treatments are distributed irregularly inside the flesh, a model based on PLS was built using the spectrum of every pixel in the fruit. The model obtained an $\mathrm{R}_{\mathrm{P}}^{2}$ of 0.91 which allowed the creation of the predicted distribution maps of the tannins in the flesh of the fruit, thereby pointing to hyperspectral systems as a promising technology to assess the effectiveness of the 
deastringency treatments that are usually applied before commercialising persimmons from astringent cultivars.

Keywords: Diospyros kaki, fruit internal quality, soluble tannins, distribution map, hyperspectral imaging, computer vision

\section{INTRODUCTION}

Astringency removal is required prior to commercialising astringent persimmon cultivars. The astringency of persimmon fruit is due to the high soluble tannin content in the flesh. Traditionally, the astringent cultivars have been consumed after fruit has been submitted to an over-ripening treatment with exogenous ethylene; under these conditions astringency removal is accompanied by a drastic loss of flesh firmness which hampers postharvest handling of the fruit. For this reason, postharvest treatments which allow astringency removal while preserving high flesh firmness have been developed in recent years. Among them, the most widely used technique in commercial settings is based on exposing fruits to high $\mathrm{CO}_{2}$ concentrations $(95 \%-98 \%)$ for $24 \mathrm{~h}$ to $36 \mathrm{~h}$. This method promotes anaerobic respiration in the fruit, giving rise to an accumulation of acetaldehyde, which reacts with the soluble tannins (ST). Tannins become insoluble at the end of the treatment and astringency is no longer detected (Matsuo \& Ito, 1982; Matsuo et al., 1991).

The optimum duration of the $\mathrm{CO}_{2}$ treatment depends on the cultivar but also on the stage of fruit maturity (Besada et al., 2010). If the treatment is too short, it may result in fruit with residual astringency, and if extended excessively it may lead to losses of fruit quality (Novillo et al., 2014). Therefore, the optimum treatment conditions must be determined for the different cultivars, but the stage of fruit maturity must also be taken into account. Therefore, the knowledge of the fruit condition at harvest according to its stages of maturity is a prerequisite to apply the adequate $\mathrm{CO}_{2}$ treatment.

After application of the $\mathrm{CO}_{2}$ treatment it is necessary to evaluate its effectiveness to avoid residual astringency that could negatively affect the future buying intentions of consumers. Currently, the effectiveness of deastringency treatment can be assessed by measuring the content of ST that remain in the flesh, although this is a slow and destructive analytical method, and thus commercially unfeasible. Another method to determine the 
level of astringency of persimmon fruit is based on the reaction of ST (responsible for astringency) with $\mathrm{FeCl}_{3}$, which leads to a blue staining; the intensity of the staining observed after a slice of the flesh is impregnated with $\mathrm{FeCl}_{3}$ depends on its level of ST. Although this method is faster and easier than the analytical determination of ST, it is also destructive and subjective and therefore it is necessary to search for new rapid, reliable, non-contact and non-destructive techniques.

Among them, computer vision represents a fast, accurate, and proved alternative for monitoring fruit quality (Cubero et al., 2011). However, this kind of sensors are limited to analysing the external properties of the fruit like colour, size or the presence of external defects, not being capable of detecting internal compounds like ST, responsible of the astringency. Therefore, other methods capable of analyse internal compounds are necessary. Spectroscopy is a non-destructive, inexpensive, rapid and reliable technique that has traditionally been used in food chemistry for qualitative and quantitative determination of different compounds in fruit samples, especially near infrared (NIR) spectroscopy (Nicolaï et al., 2007; Nicolaï et al., 2014; Magwaza et al., 2012; López et al., 2013). This technique has been utilised for the quantitative determination of soluble solids content (SSC), firmness, acidity, dry matter, chemical substances such as glucose, sucrose, citric acid, malic acid, starch or cellulose in different fruits (Schmilovitch et al., 2000; Nagle et al., 2010; Theanjumpol et al., 2013), and even to determine a maturity index (Jha et al., 2013), internal quality index (Cortés et al., 2016) or different appropriate indices for quality analysis (Attila and János, 2011).

However, a major disadvantage of spectroscopy is that only can measure in a single point of the sample. On the other hand, hyperspectral imaging is a non-destructive optical technology that integrates the advantages of spectroscopy and conventional imaging to obtain both spatial and spectral information simultaneously. It allows the visualisation of internal compounds of the fruit distributed into the image which is not possible with conventional spectroscopy (Gowen et al., 2007; Lorente et al., 2012) as in the case of the ST of the persimmon, leading to a non-valid probe measurement and forcing to take measurements in many places of the fruit surface (Noypitak et al., 2015). Thus, using hyperspectral imaging Mendoza et al., (2011) developed a method for the in-line prediction of firmness and SSC achieving coefficient of correlation between 0.83 and 0.95 for 
prediction of firmness and between 0.67 and 0.87 for prediction of SSC in different apple cultivars. Later they compared several spectral sensors to predict these properties (Mendoza et al., 2012). Cen et al. (2016) investigated the detection of internal chilling injuries in pickling cucumbers using hyperspectral reflectance $(500-675 \mathrm{~nm})$ and transmittance (675$1000 \mathrm{~nm}$ ) achieving $100 \%$ of correct detection using SVM with the fruit travelling at 100 $\mathrm{mm} / \mathrm{s}$, which gives an idea of the potential of this technology for non-destructive in-line quality control.

Ripeness has been one of the main features studied with this technology. Lleo et al. (2011) used hyperspectral imaging $(400-1000 \mathrm{~nm})$ to predict the maturity of 'Rich lady' peaches by computing different indices extracted from band ratios and combinations. The application of these indices to create maps from the classification of individual pixels showed that the ripening was not uniform throughout the entire fruit. Furthermore, the ripening of intact bell peppers was studied by Schmilovitch et al. (2014) using hyperspectral imaging $(550-850 \mathrm{~nm})$. They were able to relate some internal compounds like SSC, total chlorophyll, carotenoid and ascorbic acid content with the spectral data by means of a PLS regression, in all cases achieving an $\mathrm{R}^{2}$ higher than 0.90 except for ascorbic acid (0.72). The chemometric models they established were used to estimate internal components in each pixel of the fruit image, thus allowing mapping of the quality parameters in the intact peppers.

Apart from maturity, other properties can also be assessed. Yang et al. (2015) measured anthocyanin content in lychee pericarp in the 350-1050 nm range. They created several models achieving an $\mathrm{R}^{2}$ of 0.92 using all wavelengths. The model was later applied to entire fruits to create distribution maps with which to visualize the changes in anthocyanin content during storage time. Liu et al. (2015) used multispectral images to predict lycopene and phenolic compounds content in intact tomatoes. The comparison of methods based on PLS, least squares-support vector machines (LS-SVM) and back propagation neural networks (BPNN) showed BPNN to be the one that performed best, with an $\mathrm{R}^{2}$ of 0.96 . By applying the model to each pixel of the tomato they were able to create prediction maps of the intact tomatoes.

In persimmon fruit the use of this non-destructive technology to assess different quality parameters is beginning to be studied. Hence, Wei et al. (2014) used hyperspectral imaging 
to study the relationship between firmness and fruit maturity. Mohammadi et al. (2015) also used image analysis techniques to evaluate the index of external colour of the fruits in order to classify them into three stages of commercial maturity. Nevertheless, more studies are necessary in this regard, particularly with fruit that will be commercialized with a firm texture. Furthermore, to our knowledge, the potential of hyperspectral technology to detect the level of astringency in persimmon has not been evaluated to date. Only some works have been carried out to detect phenolic compounds related to astringency (Nogales-Bueno et al., 2014), focused especially on wine quality (Aleixandre-Tudó et al., 2015; Boulet et $a l ., 2016)$. And only one work has been found related to astringengy in persimmon using NIR spectroscopy in the range of 660-960 nm (Noypitak et al., 2015).

The aim of this work is to advance in the development of a non-destructive tool to assess the astringency of persimmon fruits that have been gone under deastringency treatment, since at present there are no methods or previous research focused on this aim for this type of fruit, being still a demand from the industry.

\section{MATERIALS AND METHODS}

\subsection{Image acquisition and calibration}

The hyperspectral imaging system capable of acquiring images in the spectral range 400-1100 nm was composed of an industrial camera (CoolSNAP ES, Photometrics, AZ, USA), and two liquid crystal tunable filters (LCTF) (Varispec VIS-07 and NIR-07, Cambridge Research \& Instrumentation, Inc., MA, USA) and a lens capable of covering the whole spectral range without losing the focus (Xenoplan 1.4/23, Schneider Optics, Hauppauge, NY, USA). The system was configured to capture images of $1392 \times 1040$ pixels with a spatial resolution of $0.14 \mathrm{~mm} /$ pixel and a spectral resolution of $10 \mathrm{~nm}$. To optimise the dynamic range of the camera, prevent saturated images and correct the spectral sensitivity of the different elements of the system, a calibration of the integration time of each band was performed by capturing the averaged reflectance of a white reference target (Spectralon 99\%, Labsphere, Inc, NH, USA) corresponding to $90 \%$ of the dynamic range of the camera. The scene was illuminated by 12 halogen spotlights of $37 \mathrm{~W}$ each (Eurostar IR Halogen MR16. Ushio America, Inc., CA, USA) powered by direct current (12 V), which lit the scene indirectly by means of diffuse reflection inside a hemispherical dome where 
whole fruits were introduced manually (Fig. 1). The inner surface of the aluminium dome was painted in white and given a rough texture using a synthetic polish sprayer in order to reduce directional reflections that could cause bright spots, thus providing highly

161 homogeneous light. A holder was used to place all samples at the same height inside the 162 dome.
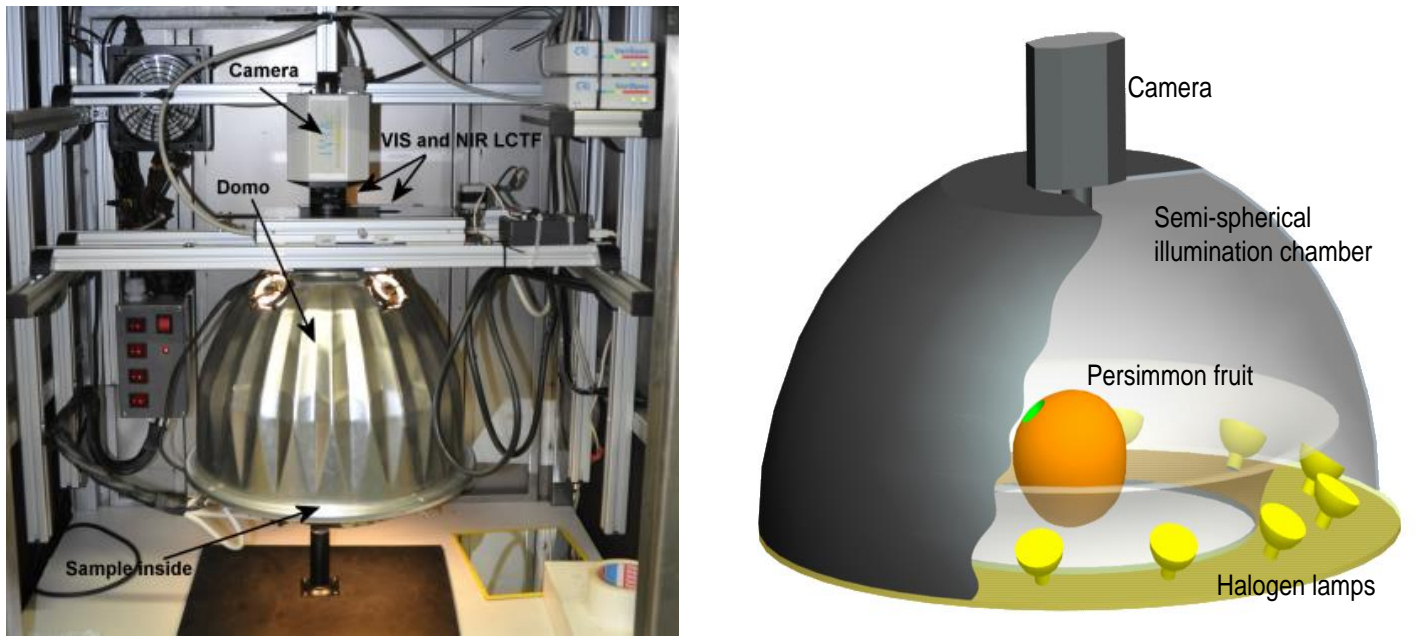

164

165

166

167

168

169

170

171

172

173

174

175

176

177

178

179

Figure 1. Configuration of the hyperspectral acquisition system

One hyperspectral image was captured of each fruit in reflectance mode in the working spectral range 460-1020 nm, which together formed a database with a total of 150 hyperspectral images with 57 wavelengths each. Equation (1) (Gat, 2000) was used to obtain the corrected relative reflectance of a pixel in the position $(x, y)$ of the monochromatic band $\lambda$.

$$
\rho_{x y}(x, y, \lambda)=\frac{\mathrm{R}^{\text {abs }}}{\mathrm{R}_{\text {white }}^{\text {abs }}}=\rho^{\mathrm{Ref}}(\lambda) \frac{\mathrm{R}(\mathrm{x}, \mathrm{y}, \lambda)-\mathrm{R}_{\text {black }}(\mathrm{x}, \mathrm{y}, \lambda)}{\mathrm{R}_{\mathrm{white}}(\mathrm{x}, \mathrm{y}, \lambda)-\mathrm{R}_{\text {black }}(\mathrm{x}, \mathrm{y}, \lambda)}
$$

where $\rho^{\operatorname{Ref}}(\lambda)$ is the standard reflectance of the white reference target (99\% in this work), $R(x, y, \lambda)$ is the reflectance of the fruit captured by the charge-coupled device (CCD) sensor of the camera, $R_{\text {white }}(x, y, \lambda)$ is the reflectance captured by the CCD of the white reference target, and $R_{\text {black }}(x, y, \lambda)$ is the reflectance captured by the CCD while avoiding any light source in order to quantify the electronic noise of the sensor. 


\subsection{Plant material and experimental design}

Persimmon cv. 'Rojo Brillante' fruits were harvested in L'Alcudia (Valencia, Spain) at three different stages of commercial maturity (M1, M2 and M3) corresponding to early and late November, and mid-December. These three stages were intentionally selected in order to obtain fruit with different degree of maturity and therefore of firmness, since the success of the treatments depend on the fruit firmness at harvest (Salvador et al., 2008) and therefore the possibility of assessing the astringency in fruits with different firmness was studied.

A total of 150 fruits with no skin damage and a homogeneous colour were selected and individually evaluated, corresponding to 50 fruits for each stage of maturity. In order to obtain different levels of astringency, fruits at each stage of maturity were divided into five homogeneous lots of 10 fruits each. Then the fruit was exposed to $\mathrm{CO}_{2}$ treatments in closed containers (air containing 95\% $\mathrm{CO}_{2}$ at $20^{\circ} \mathrm{C}$ and $90 \% \mathrm{RH}$ ) for $0 \mathrm{~h}, 6 \mathrm{~h}, 12 \mathrm{~h}, 18 \mathrm{~h}$ and $24 \mathrm{~h}$. Hyperspectral images of the intact fruits were acquired within $8 \mathrm{~h}$ after the $\mathrm{CO}_{2}$ treatment. In addition, the physicochemical parameters (external colour, firmness, ST and astringency) to achieve the objectives of this study were evaluated individually in each fruit.

\subsection{Physicochemical analysis}

The external colour index $(\mathrm{CI}=1000 a / L b)$ was measured using a colorimeter $(\mathrm{CR}-300$, Konica Minolta Inc, Tokyo, Japan) to know the colour of the fruits in each maturity stage at harvest. This index is based on the citrus colour index calculated using Hunter Lab colour values (Jiménez-Cuesta et al., 1981).

Flesh firmness was determined by means of a universal testing machine (4301, Instron Engineering Corp., MA, USA) equipped with an $8 \mathrm{~mm}$ puncture probe. The crosshead speed during testing was $1 \mathrm{~mm} / \mathrm{s}$. During the test, the force increased smoothly until it decreased drastically when the flesh was broken, and then the maximum peak force was registered. The results were expressed as the load (in N) required for breaking the flesh of the fruit on both sides after peel removal.

Analysis of variance (ANOVA) and Tukey's test were conducted to determine significant differences during maturity (colour and firmness). For this purpose, the software Statgraphics (Manugistics Corp., Rockville, Md.) was used. 


\subsubsection{Assessment of firmness}

Among the physiological changes related to fruit maturation, such as colour or decline of ST (Salvador et al., 2007), the change in fruit firmness is one of the most important

215 feature to determine the optimum postharvest condition. In this regard, Salvador et al. 216 (2008) reported that fruit firmness can influence the effectiveness of the $\mathrm{CO}_{2}$ treatment 217 because the loss of pulp structure associated to fruit softening may difficult the diffusion of $218 \mathrm{CO}_{2}$. To know if firmness could be predicted using the hyperspectral imaging system, a 219 representative ROI of the whole fruit surface was selected avoiding the stem and dark borders (Fig. 2). The average spectrum of the pixels in the ROI was calculated and

221 considered as the fruit sample. Two thirds of the fruits in each maturity stage were used to

222 build the models and the remaining fruits were used as prediction set. A pre-processing 223 Standard Normal Variate (SNV) was applied to the spectra in order to remove scatter 224 effects from the original spectral data.

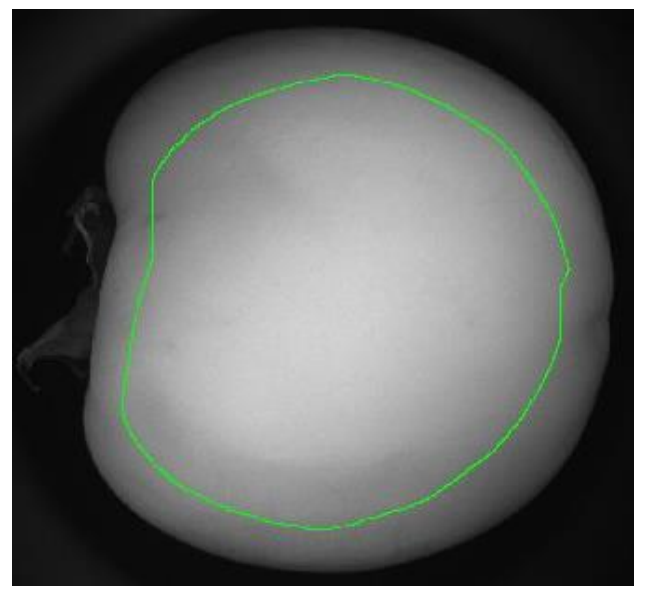

Figure 2. Hyperspectral image of a persimmon with the selected ROI (centred on $640 \mathrm{~nm}$ )

Hyperspectral systems are complex equipments that provide a huge amount of data. It is 231 using a reduced number of selected wavelengths. The Successive Projections Algorithm 232 (SPA) has been proposed as a novel variable selection strategy for multivariate calibration 233 (Araújo et al., 2001), and its purpose is to select wavelengths whose information content is 234 minimally redundant. The SPA is a forward selection method that builds an ordered chain 
of $k$ variables where each element is selected in order to present the least collinearity with the previous ones. The collinearity between variables is assessed by the correlation between the respective column vectors of the calibration matrix. As it is an iterative method, this means that several subsets containing up to $k$ variables can be formed. Finally, in order to choose the most appropriate subset, Multiple Linear Regression models are built to compare them in the terms of the RMSE. In order to select the most important bands, SPA was applied using MATLAB R2015a (The MathWorks, Inc. MA, USA) on the calibration set of fruits.

PLS-discriminant analysis (PLS-DA) was applied in order to know if it was possible to separate the fruit into the three stages of maturity at harvest using both, the entire spectrum and only the variables selected by the SPA. In a similar way, PLS regression (PLS-R) was used to predict the firmness including the data provided by the universal testing machine instead of the stages of maturity. A single 10-fold cross-validation was used to choose the optimal number of LV and accuracy and predictive capability of the PLS-R models were evaluated by RMSE and coefficient of determination $\mathrm{R}^{2}$. The software used in this study was The Unscrambler X 10.1 (CAMO Software, Oslo, Norway).

\subsubsection{Assessment of Astringency}

The level of astringency of each individual fruit was determined in three different ways:

1) Each fruit was cut in half and pressed against $10 \times 10 \mathrm{~cm}$ filter paper soaked in a $5 \%$ $\mathrm{FeCl}_{3}$ solution, obtaining a blue print whose quantity and intensity gave information about the ST content and its distribution. This process is an alternative technique to the Folin-Denis method used in industry to determine the level of astringency in fruit batches, and it has only been used to show visually the internal distribution of the ST and its intensity.

2) A flesh sample from each fruit was frozen at $-20^{\circ} \mathrm{C}$ and later the content of ST was analysed using the Folin-Denis method (Taira, 1995). The results were expressed as relative ST in fresh weight, being the quantitative reference used in our analyses.

3) The level of astringency of the fruit was evaluated by a sensory panel consisting of 10 semi-trained staff members of IVIA familiar with persimmon fruit and 
astringency attribute. Astringency was determined using a 5-point scale from 1non-astringent or absence of astringency to 5-intensely astringent.

To assess the astringency using the hyperspectral imaging system a new approach was used. As the current method used in the industry is based on the printing of the content and distribution of ST, the imaging system was used also to create maps of the distribution of the ST. Hence, the individual spectrum of each pixel in the image was used to predict the astringency of that pixel, thus using the spectral but also the spatial information provided by an image-based technology. A PLS model was built using 12 persimmons from the whole set of fruits. Six fruits (two of each maturity stage) were selected from the non-treated fruit, and thus considering as very astringent and another six (two of each maturity stage) from the batch that were treated for $24 \mathrm{~h}$.

A total of 67456 pixels labelled as astringent or non-astringent were selected from the aforementioned fruits. The spectra of these pixels were used to build the PLS model that was internally validated by means of a 10 -fold cross-validation. The model was then projected to the rest of the fruit to obtain maps of the distribution of the astringency in the fruits.

\section{RESULTS AND DISCUSSION}

\subsection{Assessment of firmness}

The colour index and the firmness of the samples at harvest are shown in Table 1. It is observed that the firmness value decreases as the fruit ripen, but in all cases studied having high firmness. In the case of the colour, the values measured indicate that fruit was changing from orange to red, as the increasing of the CI is due to an increase of the $a$ value and a reduction of $b$, both variables expressed in Hunter Lab values.

Table 1. Colour index and firmness values for the three stages of maturity at harvest

\begin{tabular}{cccc}
\hline & M1 & M2 & M3 \\
\hline CI & $9.46 \pm 1.77^{\mathrm{a}}$ & $18.20 \pm 3.32^{\mathrm{b}}$ & $21.6 \pm 4.05^{\mathrm{c}}$ \\
Firmness (N) & $43.85 \pm 4.1^{\mathrm{a}}$ & $30.8 \pm 3.5^{\mathrm{b}}$ & $24.4 \pm 4.9^{\mathrm{c}}$ \\
\hline
\end{tabular}


Values are mean \pm standard deviation. Different superscript letters in the same row indicate significant differences between groups ( $p$-value $<0.05$ ), according to Tukey's test

The SPA used to reduce the dimensionality achieved an optimal set of five wavelengths $(570,590,680,710,990 \mathrm{~nm})$. These wavelengths are associated to the range of absorption bands of carotenoids (570 and $590 \mathrm{~nm}$ ) (Choudhary et al., 2009; Merzlyak et al., 2003), chlorophyll (680 and $710 \mathrm{~nm}$ ) (Lleó et al., 2011; Rajkumar et al., 2012) and water content (990 nm) (Lu and Peng, 2006).

Table 2 shows the results obtained for the classification of persimmon fruits in terms of their stage of maturity. Both models, using all and the optimal wavelengths, achieved high percentage of fruits classified correctly (>90\%). As regarding the classification of each maturity stage, fruits of M2 were worst classified in both models.

Table 2. Maturity classification (\%) of the test set by PLS-DA using all and selected wavelengths

\begin{tabular}{ccccccc}
\hline & \#LV & Class & M1 & M2 & M3 & $\begin{array}{c}\text { Correct } \\
\text { classification }\end{array}$ \\
\hline \multirow{3}{*}{ All wavelengths } & 4 & M1 & 94.6 & 0.9 & 0.0 & \\
& & M2 & 4.0 & 91.5 & 4.4 & 93.9 \\
Selected & & M3 & 1.4 & 7.6 & 95.6 & \\
wavelengths & 2 & M1 & 92.9 & 2.7 & 0.0 & \multirow{2}{*}{ M2 } \\
& & 4.9 & 86.3 & 9.1 & \\
\hline
\end{tabular}

Table 3 shows the results of the flesh firmness prediction, obtaining similar results using all and the selected wavelengths. Values obtained were 0.77 and 0.80 for $R^{2}$ of the prediction using all and selected wavelengths, respectively. The results were not as good as the prediction results of Wei et al. (2014), who achieved an $\mathrm{R}^{2}$ value of 0.91 , but they worked with non-treated fruit having higher firmness differences between the harvest and the marketing. In the case of 'Rojo Brillante' persimmon, if they are sold as firm fruit after receiving some treatment to remove astringency, values below $10 \mathrm{~N}$ are considered as unsuitable from a commercial point of view (Salvador et al., 2004). 
Table 3. Results of firmness prediction using PLS-R with all and selected wavelengths

\begin{tabular}{cccccc}
\hline & HLV & $\mathrm{R}_{\mathrm{CV}}^{2}$ & $\mathrm{RMSE}_{\mathrm{CV}}$ & $\mathrm{R}_{\mathrm{P}}^{2}$ & $\mathrm{RMSE}_{\mathrm{P}}$ \\
\hline All wavelengths & 2 & 0.80 & 4.34 & 0.77 & 3.89 \\
Selected wavelengths & 2 & 0.80 & 4.34 & 0.80 & 3.66 \\
\hline
\end{tabular}

319

As stated, firmness is highly related to maturity, but also to astringency. As softening progresses further, acetaldehyde produced at rather low levels in the flesh also may promote tannin polymerization. Thus, in extremely soft fruit, both tannin-pectin complex formation and acetaldehyde-tannin polymerization may be involved in the reduction of astringency (Taira et al., 1997). Then, it is very important for this fruit to demonstrate that deastringency treatments do not affect the firmness.

\subsection{Astringency assessment}

The application of the different $\mathrm{CO}_{2}$ treatments resulted in fruit with a wide range of ST contents across the three stages of maturity studied, being the content of ST after different treatments between $0.75 \%$ and $0.03 \%$ (Fig. 3). This was supported by the sensory evaluation of the fruit (data not shown) that revealed that an ST content of $0.03 \%$ was related to non-astringent fruit, which is in agreement with Salvador et al., (2007) and Besada et al., (2010). ST contents higher than $0.4 \%$ led to intensely astringent fruit (sensory value of 5), while values between $0.04 \%$ and $0.4 \%$ were evaluated by the panellist as slight to medium astringency.

The evaluation of the ST by the $\mathrm{FeCl}_{3}$ method revealed a progressive reduction of the ST in the fruit as the duration treatment was longer. Moreover, it was observed that the reduction of the ST advanced from the calyx area. Figure 3 shows examples of different blue prints obtained for the different duration of the $\mathrm{CO}_{2}$ treatments.
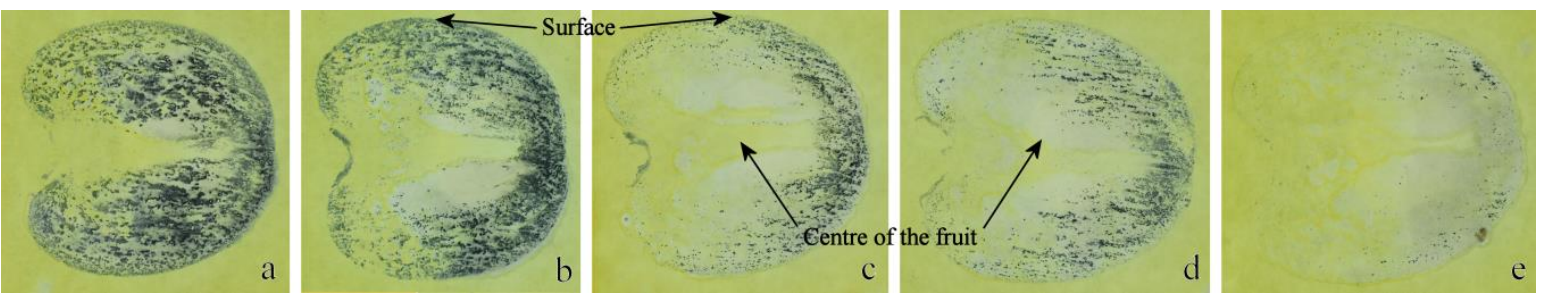
342 Figure 3. Astringency distribution from the blue print $\left(\mathrm{FeCl}_{3}\right)$ for persimmons $\mathrm{CO}_{2}$ treated 343 during different hours: a) non-treated; b-e) treated with $\mathrm{CO}_{2}$ for $6 \mathrm{~h}, 12 \mathrm{~h}, 18 \mathrm{~h}$ and $24 \mathrm{~h}$

345 The model built from astringent and non-astringent pixels using PLS was used to predict 346 the astringency of the pixels of the validation fruits and to visually compare them with the 347 prints obtained using the destructive $\mathrm{FeCl}_{3}$ method. Figure 4 shows several examples of the 348 predicted distribution map of the astringency of randomly chosen persimmons that were 349 treated with $\mathrm{CO}_{2}$ for different periods to achieve different levels of astringency (from 350 untreated fruit that was evaluated by the panellist as intensely astringent to $24 \mathrm{~h}$-treated 351 fruit, in which astringency was not detected, including medium or slight astringency for 352 fruit treated for $6 \mathrm{~h}, 12 \mathrm{~h}$ or $18 \mathrm{~h}$ ). A colour scale was used, being non-astringent pixels 353 represented by the blue colour and astringents by red colour. 


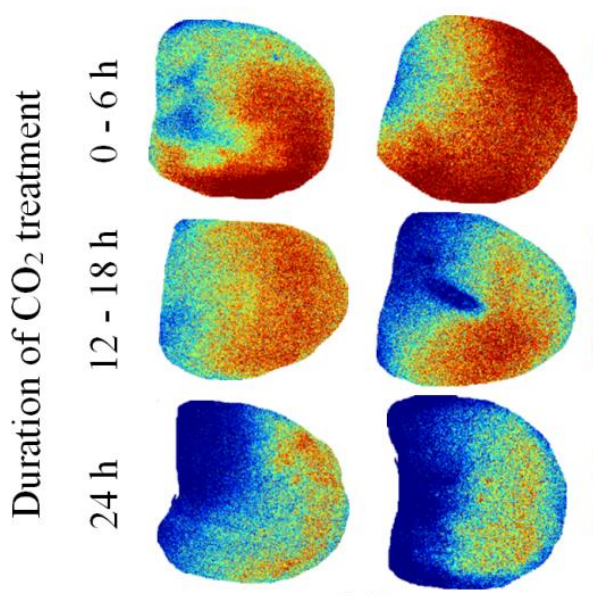

M1

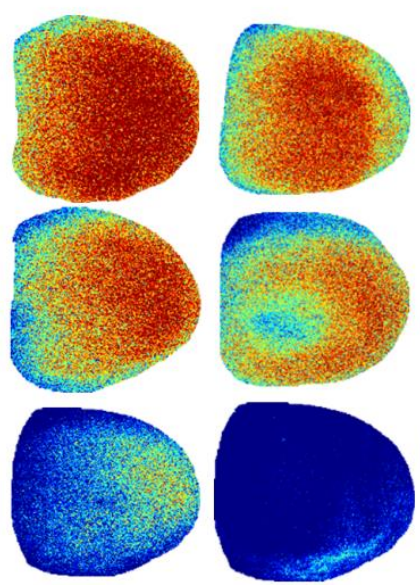

M2

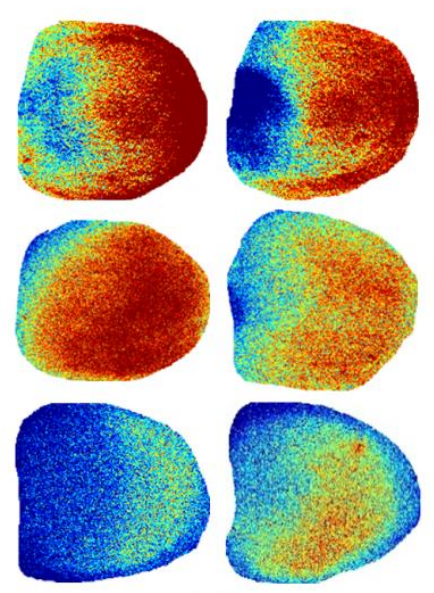

M3
357

358

359

360

361

362

363

364

365

366

367

368

369

370

371

372

373

374

375

376

Figure 4. Samples of prediction maps from hyperspectral images of persimmons in the three maturity stages

Prediction maps obtained using hyperspectral imaging system showed astringency distribution on the surface of each fruit. It was observed that the distribution of astringency in fruits under different treatments followed a very similar pattern to one obtained using destructive methods based on $\mathrm{FeCl}_{3}$ prints (Fig. 3). In Figure 4, it is clearly visible that fruits with no treatment $(0 \mathrm{~h})$ or very short $(6 \mathrm{~h})$ treatment had the highest astringency while models predicted lower astringency for fruits receiving treatment for $24 \mathrm{~h}$. In addition, it was evident in all the images that most astringency concentrated at the bottom of the fruit, while began to be removed from the calyx area, following the same trend shown in Fig. 3 .

This work contributes to lay the basis for future non-destructive tools capable to decide whether a persimmon is or not astringent in postharvest or contains residual astringency after a defective treatment, thus ensuring high quality fruit to consumers. Despite the penetration depth of the hyperspectral imaging that can be of few millimetres in the fruit, the blue prints showed that the tannins are distributed similarly in the internal part of the fruit and near the surface, and therefore can be captured by the hyperspectral images. Hence, hyperspectral imaging can be considered a valuable technique for the nondestructive evaluation of the astringency and a potential alternative to current destructive methods. 
Results achieved coincide with those achieved by Noypitak et al. (2015). They carried out a study for astringency by using spectrometry in the near-infrared zone (700-1050 nm) and measured in twelve different parts of the fruit, later calculating the average of all the measurements and obtaining results of around $\mathrm{R}^{2}$ of 0.95 . However, the authors worked with very astringent persimmons and persimmons in which all astringency was completely eliminated, avoiding the intermediate levels of astringency. Moreover, at a difference from this study, the authors used plastic bags to remove the astringency of the fruits, thereby causing a sharp loss of firmness that could have large influence the results.

\section{CONCLUSIONS}

The results of the present study showed VIS/NIR hyperspectral imaging to be a promising non-destructive tool to assess the astringency of persimmon fruits that have been gone under deastringency treatment. On one hand, the SPA method was used to select a small set of wavelengths $(570,590,680,710,990 \mathrm{~nm})$ that allowed fruit classification according to their stage of maturity using PLS-DA. The success rate obtained using the entire spectrum was slightly higher than using only the five selected wavelengths, but in both cases higher than $90 \%$. In addition, an optimum prediction of the flesh firmness was achieved using PLS regression. Moreover, using the selected wavelengths, the coefficient of determination $\mathrm{R}_{P}^{2}$ was higher (0.80) than using the full spectrum $(0.77)$.

On the other hand, it was built a predictive model that, applied to fruit submitted to deastringency treatments, allowed to create maps of the distribution of the ST that were compared visually with the traditional and subjective method based in the blue prints, to differentiate non-astringent fruits from those completely or medium astringent, thus creating a comprehensive non-destructive tool that can be used as alternative to the current destructive method based on $\mathrm{FeCl}_{3}$ prints. Therefore, hyperspectral imaging should be considered as a potential non-destructive method to evaluate the effectiveness of the deastringency treatments.

\section{ACKNOWLEDGEMENTS}

This work has been partially funded by the Instituto Nacional de Investigación y Tecnología Agraria y Alimentaria de España (INIA) through projects RTA2012-00062- 
C04-01, RTA2012-00062-C04-03 and RTA2013-00043-C02 with the support of FEDER funds and by the Conselleria d' Educació, Investigació, Cultura i Esport, Generalitat Valenciana, through the project AICO/2015/122. Sandra Munera thanks INIA for the grant FPI-INIA \#43 (CPR2014-0082) partially supported by FSE funds.

\section{REFERENCES}

Aleixandre-Tudó, J.L., Nieuwoudt, H. \& Du Toit, W.J. (2015). Robust ultraviolet-visible (UV-Vis) partial least squares (PLS) model for tannin quantification in red wine. Journal of Agricultural and Food Chemistry 63, 1088-1098.

Araújo, M. C. U., Saldanha, T. C. B., Galvão, R. K. H., Yoneyama, T., Chame, H. C. \& Visani, V. (2001). The successive projections algorithm for variable selection in spectroscopic multicomponent analysis. Chemometrics and Intelligent Laboratory Systems, 57(2), 65-73.

Attila, N. \& János, T. (2011). Sweet cherry fruit analysis with reflectance measurements. Journal Analele Universităţii din Oradea, Fascicula: Protecţia Mediului, 17, 263-270.

Besada, C., Salvador, A., Arnal, L. \& Martínez-Jávega, J.M., (2010). Optimization of the duration of deastringency treatment depending on persimmon maturity. Acta Horticulturae 858, 69-74.

Boulet, J.C., Trarieux, C., Souquet, J.M., Ducasse, M.A., Caillé, S., Samson, A., Williams, P., Doco, T. \& Cheynier, V. (2016). Models based on ultraviolet spectroscopy, polyphenols, oligosaccharides and polysaccharides for prediction of wine astringency. Food Chemistry 190, 357-363.

Cen, H., Lu, R., Zhu, Q. \& Mendoza, F. (2014). Nondestructive detection of chilling injury in cucumber fruit using hyperspectral imaging with feature selection and supervised classification. Postharvest Biology and Technology 111, 352-361.

Choudhary, R., Bowser, T. J., Weckler, P., Maness, N. O. \& McGlynn, W. (2009). Rapid estimation of lycopene concentration in watermelon and tomato puree by fiber optic visible reflectance spectroscopy. Postharvest Biology and Technology 52, 103-109. 
436 Cortés, V., Ortiz, C., Aleixos, N., Blasco, J., Cubero, S. \& Talens, P. (2016). A new 437 internal quality index for mango and its prediction by external visible and near-infrared 438 reflection spectroscopy. Postharvest Biology and Technology, 118, 148-158.

440 \& Herderich, M.J. (2004). Prediction of phenolic compounds in red wine fermentations by visible and near infrared spectroscopy. Analytica Chimica Acta 513, 73-80.

446 Gat, N. (2000). Imaging spectroscopy using tunable filters: A review. Technical report, $447 \quad$ Opto-Knowledge Systems Inc. OKSI.

Gómez-Sanchis, J., Blasco, J., Soria-Olivas, E., Lorente, D., Escandell-Montero, P., 449 Martínez-Martínez, J.M., Martínez-Sober, M. \& Aleixos, N., (2013). Hyperspectral 450 LCTF-based system for classification of decay in mandarins caused by Penicillium

Cubero, S., Aleixos, N., Moltó, E., Gómez-Sanchis, J. \& Blasco, J. (2011). Advances in machine vision applications for automatic inspection and quality evaluation of fruits and vegetables. Food Bioprocess Technology 4, 487-504.

Food Chemistry 173, 482-488 digitatum and Penicillium italicum using the most relevant bands and non-linear classifiers. Postharvest Biology and Technology 82, 76-86.

Gowen, A. A., O’Donnell, C. P., Cullen, P. J., Downey, G., \& Frias, J. M. 2007. Hyperspectral imaging - an emerging process analytical tool for food quality and safety control. Trends in Food Science and Technology 18, 590-598.

Jha, S.N., Jaiswal P, Narsaiah K, Sharma R, Bhardwaj R, Gupta M, Kumar R. (2013). Authentication of mango varieties using near infrared spectroscopy. Agricultural Research, 2(3):229 - 235. 3.

Jiménez-Cuesta MJ, Cuquerella J, Martínez-Jávega JM. (1981) Determination of a color index for citrus fruit degreening. In Proceedings of the International Society of Citriculture, Vol. 2, 750-753.

Liu, C., Liu, W., Chen, W., Yang, J. \& Zheng, L. (2015). Feasibility in multispectral imaging for predicting the content of bioactive compounds in intact tomato fruit. Food Chemistry 173, 482-488. 
Lleó, L., Roger, J.M., Herrero-Langreo, A., Diezma-Iglesias, B. \& Barreiro, P. (2011). Comparison of multispectral indexes extracted from hyperspectral images for the assessment of fruit ripening. Journal of Food Engineering 104, 612-620.

Lopez, A., Arazuri, S., Garcia, I., Mangado, J., Jaren, C. (2013) A review of the application of near-infrared spectroscopy for the analysis of potatoes. J. Agric. Food Chem., 61, $5413-5424$.

Lorente, D., Aleixos, N., Gómez-Sanchis, J., Cubero, S., García-Navarrete, O.L. \& Blasco, J., (2012). Recent advances and applications of hyperspectral imaging for fruit and vegetable quality assessment. Food Bioprocess Technology 5, 1121-1142.

Lu, R. \& Peng, Y., (2006). Hyperspectral Imaging for assessing peach fruit firmness. Biosystems Engineering 93, 161-171.

Magwaza, L.S., Opara, U.L., Nieuwoudt, H., Cronje, P.J.R., Saeys, W. \& Nicolaï, B. (2012) NIR Spectroscopy Applications for Internal and External Quality Analysis of Citrus Fruit-A Review. Food and Bioprocess Technology 5, 425-444

Matsuo, T. \& Ito, S. (1982). A model experiment for de-astringency of persimmon fruit with high carbon dioxide: in vitro gelation of kaki-tannin by reacting with acetaldehyde. Journal of Agricultural Food Chemistry 46, 683-689.

Matsuo, T., Ito, S. \& Ben-Arie, R. (1991). A model experiment for elucidating the mechanism of astringency removal in persimmon fruit using respiration inhibitors. Journal of the Japanese Society for Horticultural Science 60, 437-442.

Mendoza, F., Lu, R., Ariana, D., Cen, H. \& Bailey B. (2011). Integrated spectral and image analysis of hyperspectral scattering data for prediction of apple fruit firmness and soluble solids content. Postharvest Biology and Technology 62, 149-160.

Mendoza, F., Lu, R. \& Cen, H. (2012). Comparison and fusion of four nondestructive sensors for predicting apple fruit firmness and soluble solids content. Postharvest Biology and Technology 73, 89-98.

Merzlyak, M.N., Solovchenko, A.E. \& Gitelson, A.A. (2003). Reflectance spectral features and non-destructive estimation of chlorophyll, carotenoid and anthocyanin content in apple fruit. Postharvest Biology and Technology 27, 197-211. 
494

495

496

497

498

499

500

501

502

503

504

505

506

507

508

509

510

511

512

513

514

515

516

517

518

519

520

521

522

Mohammadi, V., Kheiralipour, K. \& Ghasemi-Varnamkhasti, M. (2015). Detecting maturity of persimmon fruit based on image processing technique. Scientia Horticulturae 184, 123-128.

Nagle, M., Mahayothee, B., Rungpichayapichet, P., Janjai, S. \& Müller, J. (2010). Effect of irrigation on near-infrared (NIR) based prediction of mango maturity. Scientia Horticulturae, 125(4), 771-774.

Nicolaï, B.M., Beullens, K., Bobelyn, E., Peirs, A., Saeys, W., Theron, K.I. \& Lammertyn, J. (2007). Non-destructive measurement of fruit and vegetable quality by means of NIR spectroscopy: A review. Postharvest Biology and Technology, 46 (2), 99-118.

Nogales-Bueno, J., Hernández-Hierro, J.M. \& Heredia. F.J. (2014). Determination of technological maturity of grapes and total phenolic compounds of grape skins in red and white cultivars during ripening by near infrared hyperspectral image: a preliminary approach. Food Chemistry 152, 586-591.

Novillo, P., Salvador, A., Llorca, E., Hernando, I. \& Besada, C. (2014). Effect of CO2 deastringency treatment on flesh disorders induced by mechanical damage in persimmon. Biochemical and microstructural studies. Food Chemistry 145, 453 - 463.

Noypitak, S., Terdwongworakul, A., Krisanapook, K. \& Kasemsumran, S. (2015). Evaluation of astringency and tannin content in 'Xichu' persimmons using near infrared spectroscopy. International Journal of Food Properties, 18:1014-1028.

Salvador, A., Arnal, L., Besada, C., Larrea, V., Quiles, A. \& Pérez-Munuera, I. (2007). Physiological and structural changes during ripening and deastringency treatment of persimmon cv. 'Rojo Brillante'. Postharvest Biology and Technology 46, 181-188.

Salvador, A., Arnal, L., Besada, C., Larrea, V., Quiles, A. \& Pérez-Munuera, I. (2008). Reduced effectiveness of the treatment for removing astringency in persimmon fruit when stored at $15^{\circ} \mathrm{C}$. Physiological and microstructural study. Postharvest Biology and Technology 49, $340-347$.

Salvador, A., Arnal, L., Carot, J.M.., Carvalho, C.P.. \& Jabaloyesm, J.M. (2006). Influence of different factor son firmness and color evolution during the storage of persimmon cv. 'Rojo Brillante'. Sensory and Nutritive Qualities of Food, 71(2), 170-175. 
Salvador, A., Arnal, L., Monterde, A. \& Cuquerella, J. (2004). Reduction of chilling injury symptoms in persimmon fruit cv 'Rojo brillante' by 1-MCP. Postharvest Biology and Technology 33, 285-291.

Schmilovitch, Z., Ignat, T., Alchanatis, V., Gatker, J., Ostrovsky, V. \& Felföldi, J. (2014). Hyperspectral imaging of intact bell peppers. Biosystems engineering Special Issue: Image Analysis in Agriculture 117, 83-93.

Schmilovitch, Z., Mizrach, A., Hoffman, A., Egozi, H. \& Fuchs, Y. (2000). Determination of mango physiological indices by near-infrared spectrometry. Postharvest Biology and Technology, 19(3), 245-252.

Taira, S. (1995). Astringency in persimmon. In: Linskens, H.F., Jackson, J.F. Fruit Analysis. Springer, Hannover, Germany, pp. 97-110.

Taira, S., Ono, M. \& Matsumoto N. (1997) Reduction of persimmon astringency by complex formation between pectin and tannins. Postharvest Biology and Technology 12, 265-271

Theanjumpol, P., Self, G., Rittiron, R., Pankasemsu, T., \& Sardsud, V. (2013). Selecting Variables for Near Infrared Spectroscopy (NIRS) Evaluation of Mango Fruit Quality. Journal of Agricultural Science, 5(7).

Vélez-Rivera, N., Blasco, J., Chanona-Pérez, J., Calderón-Domínguez, G., Perea-Flores, M.J., Arzate-Vázquez, I., Cubero, S. \& Farrera-Rebollo, T. (2014). Computer vision system applied to classification of "Manila" mangoes during ripening process. Food and Bioprocess Technology 7, 1183-1194.

Wei, X., Liu, F., Qiu, Z., Shao, Y. \& He, Y. (2014). Ripeness Classification of Astringent Persimmon Using Hyperspectral Imaging Technique. Food and Bioprocess Technology 7, 1371-1380.

Yahaya, O.K.M., MatJafri, M.Z., Aziz, A.A. \& Omar, A.F. (2015). Visible spectroscopy calibration transfer model in determining $\mathrm{pH}$ of Sala mangoes. Journal of Instrumentation, 10, T05002.

Yang, Y.C., Sun, D.-W. Pu, H., Wang, N.N. \& Zhu, Z. (2015). Rapid detection of anthocyanin content in lychee pericarp during storage using hyperspectral imaging coupled with model fusion. Postharvest Biology and Technology 103, 55-65. 